\title{
Impacto de Una Política Pública En La Disminución de Las Lesiones Y La Mortalidad Por Accidentes de Tráfico Atribuibles Al Alcohol
}

\author{
1Jose D Charry, ${ }^{2}$ Maria C Erazo, ${ }^{3}$ Marly Y Vargas, ${ }^{4}$ Juan C Puyana, ${ }^{5}$ Andres M Rubiano
}

\section{RESUMEN}

Introducción: El trauma es considerado un problema de salud pública. Es una de las patologías que generan mayor discapacidad y mortalidad a nivel de Latinoamérica. El objetivo de este estudio fue evaluar los ingresos de pacientes politraumatizados en un hospital universitario de Colombia antes y después de la implementación de una política pública que aumentó la vigilancia y las penalidades asociadas a conducir en estado de embriaguez.

Materiales y métodos: Se construyó una base de datos de pacientes politraumatizados que ingresaron entre junio de 2013 y abril de 2014, con antecedentes de ingesta de alcohol. Se determinó el resultado de acuerdo al tiempo de ingreso con respecto a la implementación de la política pública y se realizó un análisis univariado. Se calcularon medidas de tendencia central y dispersión para las variables continuas.

Resultados: En total se analizaron 1295 pacientes, 760 antes y 535 después de la implementación de la política. La mediana de la edad fue de 34,6 vs 34,4 p: 0,793. La mediana del ISS para los grupos fue de 25 vs 24.8 p: 0,976. Con la implementación de la política pública, los ingresos hospitalarios disminuyeron de un $58,7 \%$ vs $41,3 \%$ p: 0,001 y la mortalidad en el servicio de urgencias disminuyó de 5,13\% a 2,42\% p: 0,003.

Conclusión: La adopción de una política pública que aumentó la vigilancia y las penalidades por conducir en estado de embriaguez, está asociada a la disminución de los ingresos hospitalarios y de la mortalidad en el servicio de urgencias en un hospital universitario de Colombia.

Palabras claves: Política pública, Mortalidad, Accidentes de tráfico, Alcohol.

How to cite this article: Charry JD, Erazo MC, Vargas MY, Puyana JC, Rubiano AM. Impacto de Una Política Pública En La Disminución de Las Lesiones Y La Mortalidad Por Accidentes de Tráfico Atribuibles Al Alcohol. Panam J Trauma Crit Care Emerg Surg 2014;3(3):101-104.

\footnotetext{
${ }^{1-3}$ Medical Student, ${ }^{4,5}$ Professor

${ }^{1-3}$ Facultad de Salud, Universidad Surcolombiana, Colombia

${ }^{4}$ Department of Surgery, University of Pittsburgh, Pennsylvania USA
}

${ }^{5}$ Department of Surgery, Universidad Surcolombiana, Colombia

Corresponding Author: Jose D Charry, Medical Student Facultad de Salud, Universidad Surcolombiana, Colombia Phone: 3158019678, e-mail: danielcharry06@gmail.com

\section{Source of support: Nil}

Conflict of interest: None

\section{ABSTRACT}

Introduction: Trauma is considered a public health problem. It is a disease generating important disability and mortality in Latin America. The aim of this study was to assess the trend of trauma patients in a university hospital in Colombia before and after implementation of a public policy that increased surveillance and penalties associated with drunk driving.

Materials and methods: A database of trauma patients admitted between June 2013 and April 2014, with a history of alcohol intake was build. The results according to the admission time with respect to the period of implementation of the public policy were analyzed and a univariate analysis was performed. Measures of central tendency and dispersion for continuous variables were calculated.

Results: A total of 1295 patients were analyzed, 760 before and 535 after implementation of the policy. The median age was 34.6 vs 34.4 p: 0,793. The median ISS for the groups was 25 vs $24.8 \mathrm{p}$ : 0,976. With the implementation of public policy, hospital admissions decreased from $58.7 \%$ vs $41.3 \%$ p: 0,001 and mortality in the ED decreased from $5.13 \%$ to $2.42 \%$ p: 0,003 .

Conclusion: The adoption of a public policy that increased surveillance and penalties for driving while intoxicated, is associated with a decrease in hospital admissions and mortality in the emergency department at a university hospital in Colombia.

Keywords: Public policy, Mortality, Traffic accidents, Alcohol.

\section{INTRODUCCIÓN}

El trauma es una patología de importancia a nivel global. Genera alrededor de 5 millones de muertes al año, de las cuales 1,2 millones son por accidentes de tráfico., ${ }^{1,2}$ De acuerdo al estudio de la Organización Mundial de la Salud, sobre carga global de enfermedad publicado en el año 2010, ${ }^{3,4}$ el trauma continúa siendo un problema de salud pública y genera una carga importante para los sistemas de salud en países latinoamericanos; en Colombia la carga global de las lesiones, está más marcada en la población económicamente activa de sexo masculino entre 12 y 45 años; en el 2013 por ejemplo, se presentaron alrededor de 48.000 casos de accidentes de transporte; para un total de 6.219 personas fallecidas y gran mayoría de ellas estaban asociadas a alcohol. ${ }^{5}$ 
El objetivo de este estudio fue evaluar los ingresos de pacientes politraumatizados en un hospital universitario de Colombia antes y después de la implementación de una política pública que aumento la vigilancia y las penalidades asociadas a conducir en estado de embriaguez.

\section{MATERIALES Y MÉTODOS}

\section{Diseño}

Estudio retrospectivo observacional de los pacientes politraumatizados victimas de lesiones por accidente de tráfico y que fueron atendidos en el Hospital Universitario de Neiva entre junio de 2013 y abril de 2014.

Se evaluaron las variables y se determinó el resultado de acuerdo al tiempo de ingreso al servicio de urgencia del hospital Universitario de Neiva, con respecto a la implementación de la política pública, generando dos grupos. El primer grupo de pacientes corresponde a los ingresos hospitalarios previos a la implementación de la política pública (1 Junio de 2013 a 30 de noviembre de 2013) y el segundo grupo es posterior a la implementación de la política pública (1 de diciembre de 2013 a 30 de abril de 2014).

\section{Criterio de Inclusión y Exclusión}

Fueron incluidos en el estudio aquellos pacientes politraumatizados mayores de 18 años que ingresaron a la institución con antecedentes de ingesta de alcohol.

\section{Recolección de Datos y Análisis Estadístico}

El método usado para la recolección de datos fue observacional directo no participativo. Se realizó la revisión documental de las historias clínicas registrando los datos de esta en un formulario con datos epidemiológicos, clínicos y sociales. Los resultados obtenidos en el estudio fueron almacenados y analizados en un software estadístico en línea $\mathrm{R}$ versión 2.15.2; Se calcularon medidas de tendencia central y dispersión para las variables continuas; así como frecuencias y proporciones para las categóricas.

\section{Políticas de Control de Alcohol}

En Colombia existen diferentes políticas que controlan y regulan el consumo y venta del alcohol. Sin embargo el alto número de lesiones atribuibles al alcohol, hizo pensar en crear una nueva política pública. Esta nueva ley se caracterizó por el aumento en la vigilancia de las personas que consumen alcohol estableciendo zonas permitidas para el consumo y venta de alcohol con el objetivo de poder tener puestos de vigilancia por las autoridades competentes, las restricciones y las penalidades asociadas a conducir en estado de embriaguez. Estas penalidades se caracterizaron por: (1. Persona conduciendo bajo estado de embriaguez tendrían como penalidad multas de elevado costo económico, 2. Persona conduciendo bajo estado de embriaguez que causen algún accidente de tráfico sin lesiones mayores se sancionaría con la suspensión o cancelación de la licencia de conducción hasta por 10 años, 3. Personas conduciendo bajo estado de embriaguez que cause la muerte de alguna persona tendría como sanción inmediata la suspensión definitiva de la licencia de conducción y procesos penales). Esta ley fue implementada en diciembre de 2013.

\section{RESULTADOS}

En total se analizaron 1295 pacientes, 760 antes y 535 después de la implementación de la política entre junio de 2013 y abril de 2014, La mediana de la edad fue de 34,6 vs 34,4. La mediana del ISS para los grupos fue de 25 vs 24.8. Con la implementación de la política pública, los ingresos hospitalarios disminuyeron de un 58,7\% vs 41,3\%. Las características Clinicas y sociodemográficas de la población total se describen en la (Tabla 1).

De los pacientes que ingresaron a la institución con lesiones atribuibles al alcohol, los diagnósticos más frecuentes fueron politraumatismo, trauma craneoencefálico (TCE) y trauma de tórax. Se describen en la Tabla 2. El mecanismo del trauma contundente fue observado en $93.4 \%$ para el grupo pre implementación y en $97,2 \%$ en el grupo de paciente pos implementación. Las heridas penetrantes se evidenciaron en $6.6 \%$ de los pacientes del grupo pre implementación de la política pública y en 2,8\% para el grupo pos implementación. La mortalidad en el servicio de urgencias disminuyó de $39(5,13 \%)$ a $13(2,42 \%)$. En la Tabla 3, se describe el tipo

Tabla 1: Características clínicas y sociodemográficas de los pacientes que ingresaron pre y pos Implementación de la Política Pública

\begin{tabular}{llll}
\hline & $\begin{array}{l}\text { Pre } \\
\text { implementación } \\
\text { N:760 }\end{array}$ & $\begin{array}{l}\text { Pos } \\
\text { implementación } \\
\text { N:535 }\end{array}$ & $p$ \\
\hline $\begin{array}{l}\text { Variable } \\
\text { Género }\end{array}$ & & \\
- Masculino & $551(72,5 \%)$ & $362(67,7 \%)$ & 0,0692 \\
- Femenino & $209(27,5 \%)$ & $173(32,3 \%)$ & \\
$\begin{array}{l}\text { Edad (años) } \\
\text { - Mediana (DE) }\end{array}$ & $34,6 \pm 14,20$ & $34,4 \pm 16,05$ & 0.7939 \\
- Rango & $(18-48)$ & $(18-50)$ & \\
$\begin{array}{l}\text { Glasgow (Ingreso) } \\
\text { - Media x (DE) }\end{array}$ & $6,6 \pm 3,54$ & $6,3 \pm 4,8$ & 0.9333 \\
$\begin{array}{l}\text { ISS } \\
\text { - Mediana (DE) }\end{array}$ & $25 \pm 3,96$ & $24,8 \pm 11,10$ & 0.9763 \\
\hline
\end{tabular}

Fuente: Base de datos de pacientes del Hospital Universitario de Neiva. (DE) Desviacion estándar 
Tabla 2: Hallazgos clínicos de los pacientes que ingresaron pre y pos Implementación de la Política Pública

\begin{tabular}{llll}
\hline & $\begin{array}{l}\text { Pre imple- } \\
\text { mentación } \\
\text { N:760 }\end{array}$ & $\begin{array}{l}\text { Pos imple- } \\
\text { mentación } \\
\text { N:535 }\end{array}$ & $p$ \\
\hline Variable & & & \\
Diagnostico & & & \\
- Politraumatismo & $268(35,3 \%)$ & $200(37,4 \%)$ & 0.0017 \\
- TCE severo & $261(34,3 \%)$ & $117(21,9 \%)$ & 0,0001 \\
- Trauma de Toráx & $104(13,7 \%)$ & $99(18,5 \%)$ & 0.7256 \\
- Trauma de Abdomen & $127(16,7 \%)$ & $119(22,2 \%)$ & 0.6099 \\
Resultado Final & & & \\
- Vivo & $708(93,2 \%)$ & $515(96,3 \%)$ & 0.0001 \\
- Muerto & $52(6,8 \%)$ & $20(3,7 \%)$ & \\
\hline
\end{tabular}

Fuente: Base de datos de pacientes del Hospital Universitario de Neiva

de vehículo relacionado con el mecanismo de lesión del paciente.

El promedio del tiempo de la estancia hospitalaria en el grupo de pacientes pre implementación de la política pública fueron $16,6 \pm 5,78$ días; mientras que en el grupo de pacientes pos implementación de la política pública, la estancia hospitalaria fue de 12,3 días.

\section{DISCUSIÓN}

El trauma es una de las patologías que causan mayor discapacidad y mortalidad a nivel global, genera alrededor de 5 millones de muertes al año, afecta a la personas entre las edades de 14 a 45 años las cuales son población jóvenes y más productivas desde el punto de vista laboral. ${ }^{6,7}$ En nuestro estudio observamos que la tendencia en las edades se mantiene entre los 18 y 48 años con una mediana de 34 años.

El trauma es considerado un problema de salud pública. La mortalidad ha sido tradicionalmente el principal indicador de la magnitud de un problema de salud pública, pero es importante destacar que por cada muerte, hay miles de sobrevivientes que quedan con secuelas físicas y emocionales. El trauma no solamente afecta a la víctima sino también a sus familias, comunidades y a la sociedad en general. Debido a que la organización mundial de la salud OMS considera al trauma como una epidemia, se han conformado diferentes centros especializados en el manejo de los pacientes víctima del trauma con el objetivo de disminuir mortalidad. ${ }^{8,9}$ La determinación del estado de embriaguez es una rutina en este tipo de instituciones ya que existen políticas públicas que obligan a evaluar a todos las víctimas comprometidas en accidentes de tránsito y/ó situaciones de violencia social.

En nuestro estudio encontramos que las lesiones relacionadas con el uso del alcohol son más comunes en pacientes del género masculino como se ha descrito en muchos estudios. ${ }^{10-12}$ En cuanto al mecanismo del trauma
Tabla 3: Tipo del vehículo relacionado con la atención de los pacientes que ingresaron pre y pos Implementación de la Política Pública

\begin{tabular}{llll}
\hline & $\begin{array}{l}\text { Pre } \\
\text { implementación } \\
\text { N:760 }\end{array}$ & $\begin{array}{l}\text { Pos } \\
\text { implementación } \\
\text { N:535 }\end{array}$ & $p$ \\
\hline $\begin{array}{l}\text { Variable } \\
\text { Tipo de Vehículo } \\
\text { - Motocicleta }\end{array}$ & $365(48 \%)$ & $260(48,6 \%)$ & 0.0001 \\
- Bicicleta & $91(11,9 \%)$ & $87(16,3 \%)$ & 0.7643 \\
- Vehículo & $195(25,7 \%)$ & $99(18,5 \%)$ & 0.0001 \\
& Liviano \\
- Camión/ & $101(13,3 \%)$ & $69(12,9 \%)$ & 0.0141 \\
$\quad \begin{array}{l}\text { Camioneta/ } \\
\quad \text { Bus }\end{array}$ & & & \\
- Otro & $8(1,1 \%)$ & $20(3,7 \%)$ & 0.0233 \\
\hline
\end{tabular}

${ }^{*}$ Fuente: Base de datos de pacientes del Hospital Universitario de Neiva

más frecuentemente observado, el trauma contundente se presentó en un $93.4 \%$ para el grupo pre implementación y en $97,2 \%$ en el grupo de paciente pos implementación, similar a lo descrito en otros estudios. ${ }^{13,14} \mathrm{El}$ área anatómica más comúnmente comprometidas fue la craneoencefálica. Las clasificadas como traumatismos múltiples fueron igualmente de alta incidencia. Esto también continúa siendo un patrón común descrito en otras publicaciones del mismo tema. ${ }^{15-17}$

En Colombia hasta el mes de diciembre del año 2013, se aumentaron las penalidades legales para los conductores que conducen bajo estado de embriaguez. A pesar que en nuestro estudio se evidenció una clara disminución de la afluencia de pacientes luego de la implementación de la política pública, es importante comparar las tendencias que se han mostrado desde hace varios años, donde para el año 2013 el análisis de estas tendencias evidencia el aumento de víctimas mortales en $1,09 \%$ con respecto a las cifras consolidadas de 2012 y un aumento de 13,42\% con respecto a cifras promedio de la última década. ${ }^{5}$ Aunque para el 2014, existe una tendencia a la disminución de los ingresos, es claro que si este tipo de intervenciones no se sostienen sobre el tiempo y no hay continuidad en el proceso de control, existe la posibilidad de que las cifras vuelvan a un ascenso. A pesar que existen múltiples posibles explicaciones para la disminución de estos casos, nosotros consideramos que probablemente esta medida pudo estar asociada a la disminución de los ingresos hospitalarios de pacientes politraumatizados en nuestra institución.

\section{CONCLUSIONES}

La adopción de una política pública que aumentó la vigilancia y las penalidades por conducir en estado de embriaguez, puede estar asociada a la disminución de los 
ingresos hospitalarios y de la mortalidad en el servicio de urgencias en un hospital universitario de Colombia.

\section{REFERENCIAS}

1. Byass P, de Courten M, Graham WJ, Laflamme L, McCawBinns A, et al. Reflections on the global burden of disease 2010 estimates. PLoS Medicine 2013;10(7):e1001477.

2. Horton R. GBD 2010: understanding disease, injury, and risk. Lancet 2012 Dec 15;380(9859):2053-2054.

3. Norton R, Kobusingye O. Injuries. N Engl J Med 2013 May 2;368(18):1723-1730.

4. Chisholm D, Rehm J, M VO, Monteiro M. Reducing the global burden of hazardous alcohol use: a comparative costeffectiveness analysis. J Stud Alcohol (Internet) Nov 1;65(6): 782-793.

5. Vargas DA. Comportamiento de muertes y lesiones por accidente de transporte, Colombia, 2013. Forensis, 2013. p. 171-220.

6. Medina E, Kaempffer A. Consideraciones epidemiológicas sobre los traumatismos en chile. Rev Chilena de Cirugía 2007;59(3):175-218.

7. Ordoñez C, Pino L, Tejada J, Badiel M, Gutierrez M, et al. Experencia en dos hospitales de tercer nivel de atención del suroccidente de Colombia en la aplicación del registro internacional de trauma de la sociedad panamericana de trauma (RIT/SPT-ITSDP). Rev Col Bras Cir 2012;34(4): 255-262.

8. Cheng-Min H, Lunnen J, Miranda J, Hyder A. Traumatismos causado por el tránsito en países en desarrollo: Agenda de investigación y acción. Rev Peru Med Exp Salus Pública 2010;27(2):243-247.
9. Briceno-Leon R, Villaveces A, Concha-Eastman A. Understanding the uneven distribution of the incidence of homicide in Latin America. Int J Epidemiol 2008 Aug;37:751-757.

10. Rehm J, Room R, Monteiro M, Gmel Graham K, Rehn N, et al. alcohol as a risk factor for global burden of disease. Eur Addict Res (Internet) 2003 Octubre 1;9(4):157-164.

11. Pridemore WA, Chamlin MB. A time-series analysis of the impact of heavy drinking on homicide and suicide mortality in Russia, 1956-2002. Addiction 2006 Dec;101(12):1719-1729.

12. Klimkiewicz A, Jakubczyk A, Wnorowska A, Klimkiewicz J, Bohnert A, Ilgen MA, et al. Violent behavior and driving under the influence of alcohol: prevalence and association with impulsivity among individuals in treatment for alcohol dependence in Poland. Eur Addict Res 2014;20(3):151-158.

13. Saar I. Do alcohol excise taxes affect traffic accidents? evidence from estonia. Traffic Inj Prev 2014 Jun 20:0.

14. Ju Y, Sohn SY. Time to death analysis of road traffic accidents in relation to delta $\mathrm{V}$, drunk driving, and restraint systems. Traffic Inj Prev 2014;15(8):771-777.

15. Hsieh CH, Su LT, Wang YC, Fu CY, Lo HC, Lin CH. Does alcohol intoxication protect patients from severe injury and reduce hospital mortality? The association of alcohol consumption with the severity of injury and survival in trauma patients. Am Surg 2013 Dec;79(12):1289-1294.

16. Sánchez AI, Villaveces A, Krafty RT, Park T, Weiss HB, Fabio A, et al. Policies for alcohol restriction and their association with interpersonal violence: a time-series analysis of homicides in Cali, Colombia. Int J Epidemiol 2011 Aug; 40(4):1037-1046.

17. Villaveces A, Cummings P, Koepsell TD, Rivara FP, Lumley T, Moffat J. Association of alcohol-related laws with deaths due to motor vehicle and motorcycle crashes in the United States, 1980-1997. Am J Epidemiol 2003 Jan 15;157(2):131-140. 